\title{
Polarization Effects in the Front End of the Neutrino Factory
}

R. C. Fernow and J.C. Gallardo

Brookhaven National Laboratory, Upton, New York

Y. Fukui

UCLA, Los Angeles, California

September 2000 


\title{
Polarization effects in the Front End of the Neutrino Factory
}

\author{
R.C. Fernow \& J.C. Gallardo \\ Physics Department, Brookhaven National Laboratory, Upton, NY 11973-5000 \\ Y. Fukui \\ Department of Physics and Astronomy, Box 951547, University of California, \\ Los Angeles, CA 90095-1547
}

\begin{abstract}
We summarize the methods used for simulation of polarization effects in the front end of a possible neutrino factory. We first discuss the helicity of muons in the pion decay process. We find that, neglecting acceptance considerations, the average helicity asymptotically approaches a magnitude of 0.185 at large pion momenta. Next we describe the methods used for tracking the spin through the complicated electromagnetic field configurations in the front end of the neutrino factory, including rf phase rotation and ionization cooling channels. Various depolarizing effects in matter are then considered, including multiple Coulomb scattering and elastic scattering from atomic electrons. Finally, we include all these effects in a simulation of a $480 \mathrm{~m}$ long, double phase rotation front end scenario.
\end{abstract}

\section{Introduction}

A polarized muon beam should provide a valuable handle for understanding the physics at a neutrino factory since the polarization has a strong effect on the angular distributions of the neutrinos coming from the muon decay [1]. In a similar manner polarization information is also thought to be valuable for understanding the physics in a muon collider [2].

Muon beam polarization is normally described in terms of the helicity of each muon, which is defined to be the projection of the spin vector $\mathbf{s}$ in the muon rest frame along its momentum $\mathbf{p}$. The spin vector is normalized to 1 in the muon rest frame. By convention [3] the $\mu^{+}$has helicity $=-1$ in the $\pi^{+}$rest frame. However, in the transformation of the muon into the LAB frame an 


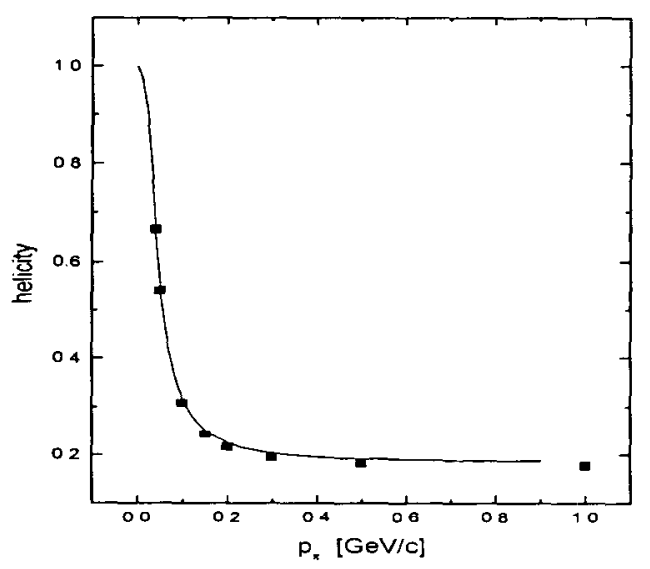

Fig. 1. Magnitude of the average muon helicity in the LAB frame as a function of pion momentum for an ideal pion beam and neglecting acceptance considerations.

angle develops between the transformed muon's momentum and its spin in the rest frame. As a result the magnitude of the helicity in the LAB frame is generally less than 1 and consequently the muon beam polarization is less than 1.

After the muon has been created it must propagate through a maze of complicated electric and magnetic fields in the front end of a neutrino factory or muon collider. Since the spin evolution follows a different set of equations than the momentum, small depolarization effects can be introduced that reducc the average polarization of the muon beam. In addition the cooling channels contain substantial amounts of material in the absorbers, absorber windows, and $r f$ cavity windows if pillbox cavities are used. Large angle Coulomb scattering and elastic scattering from atomic electrons have a finite probability of flipping the muon spin direction and can thus lead to further depolarization of the muon beam. Many additional details concerning these calcualtions can be found in a previous technical note [4].

\section{Muon polarization in pion decay}

Expressions for the muon helicity in the LAB frame can be found in the literature [5-7] as a function of the production angle in the pion rest frame. Fig. 1 shows the variation of the average muon helicity in the LAB frame as a function of the pion momentum. The solid curve is from a calculation using the Wigner formalism, while the points are from the simulation code ICOOL [8], which uses the Hayakawa expression [5].

The ICOOL simulation used a monochromatic pion beam with 0 transverse 
emittance. We averaged the helicity for 10000 random pion decays. We see that both simulations agree and that the helicity has the value 1 as it should at very low pion momentum. When the average helicity function shown in Fig. 1 is convoluted with the accepted muon momentum spectrum for typical muon collider scenarios [9], the average polarization of the accepted muon beam is typically $\sim 20 \%$. As the pion momentum increases, the helicity falls rapidly and approaches an asymptotic value given by the expression

$$
\langle h\rangle \Rightarrow \frac{1}{2}\left(1-\frac{E^{* 2}}{p^{* 2}}\right) \ln \left[\frac{E^{*}+p^{*}}{E^{*}-p^{*}}\right]+\frac{E^{*}}{p^{*}}=0.185
$$

where $p^{*}=30 \mathrm{MeV} / \mathrm{c}$ and $E^{*}=110 \mathrm{MeV}$ refer to the muon momentum and energy in the pion rest frame.

\section{Spin tracking}

When spin tracking is required each muon needs an associated 3-component, normalized spin vector in the muon rest frame. We start with the known spin vector in the pion rest frame. The spin vector is then boosted to the LAB frame using the pion properties and the appropriate Lorentz transformation. A second transformation can then be made to the muon rest frame $[5,10]$.

The muon spin vector in the rest frame evolves in electric and magnetic fields according to the Thomas-BMT equation $[10,11]$. This differs from the equation of motion for the momentum vector because of additional terms proportional to the g-factor anomaly for the muon, which has the value $1.165 \times 10^{-3}$.

As a test of likely depolarization effects in the neutrino factory due to the differing evolution equations, we examined the spin tracking in an early example of the double phase rotation front end scenario [12]. This example uses a pion input beam file made from the interactions of $24 \mathrm{GeV} / \mathrm{c}$ protons on a mercury target. There is a solenoidal field of $20 \mathrm{~T}$ around the target that tapers down to $1.25 \mathrm{~T}$ over a distance of about $8 \mathrm{~m}$ and then continues at that value over the $45 \mathrm{~m}$ long $r f$ phase rotation channel. The actual phase rotation is done with a sequence of $r f$ cavities that vary in frequency from 30 to $45 \mathrm{MHz}$ and that have electric field gradients up to $6 \mathrm{MV} / \mathrm{m}$. There were no windows or other materials in the muon path in the phase rotation channel. The phase rotation is followed by a $1.8 \mathrm{~m}$ long liquid hydrogen minicooler and then a 10 $\mathrm{m}$ long field reversal region where the solenoid field changes from $+1.25 \mathrm{~T}$ to $-1.25 \mathrm{~T}$. No significant changes in helicity were found in the regions of rapidly varying magnetic field or in the $r f$ cavities.

When the muons were propagated through the phase rotation system with spin 
tracking turned off we found that the average helicity of all muons accepted in the channel at the entrance to the minicooler is $-0.189 \pm 0.010$. There is no material in the beam up to this point. With spin tracking enabled the average helicity of the accepted muons drops slightly to $-0.181 \pm 0.010$. A special run with the g-factor anomaly set to 0 gave $-0.188 \pm 0.010$, so most of the deviation can be attributed to the extra terms in the spin equation of motion.

\section{Depolarization effects in matter}

Each scattering of a muon in matter has a finite probability of changing the muon's spin direction. This in turn can lead to a depolarization of the muon beam. We have considered two possible depolarizing processes, elastic and multiple Coulomb scattering. Both of these processes change the momentum vector of the muon. Our approach is to first change the spin vector in each interaction in such a way that the helicity is conserved. Then, using the appropriate kinematics for the event, we determine the quantum mechanical probability for the helicity to flip in the interaction. We then randomly flip muon spin directions with that probability.

Continuous energy loss (Bethe-Bloch) is another important process that is simulated for particles in matter. However, in the continuous energy loss process the code scales the magnitude of the momentum vector to correspond to the reduced kinetic energy available after a step. The direction of the momentum is unchanged in this process, and thus the helicity is not changed by it.

The tracking algorithm conserves helicity in scattering events by first computing the helicity $h$ before each scattering. Then, if primes refer to quantities after the scattering, the spin vector after the scattering is taken as

$$
\hat{s^{\prime}}=h \hat{p} \prime+\sqrt{1-h^{2}} \frac{(\hat{p} \prime \times \hat{s}) \times \hat{p} \prime}{\mid(\hat{p} \prime \times \hat{s}) \times \hat{p} \prime}
$$

As a test of the effectiveness of this algorithm, we show in Fig. 2 the behavior of the helicity in the $1.8 \mathrm{~m}$ long liquid hydrogen minicooler. The helicity remains approximately constant, even though numerous scattering events occur and the kinetic energy drops by a large amount.

Norum \& Rossmanith [13] have given an approximate estimate of the spin flip probability based on the non-relativistic calculation of the spin flip probability by Ford \& Mullin [14]. The calculated spin flip probability in multiple Coulomb scattering is known to be very small $[15-17,14]$. We have computed the spin 


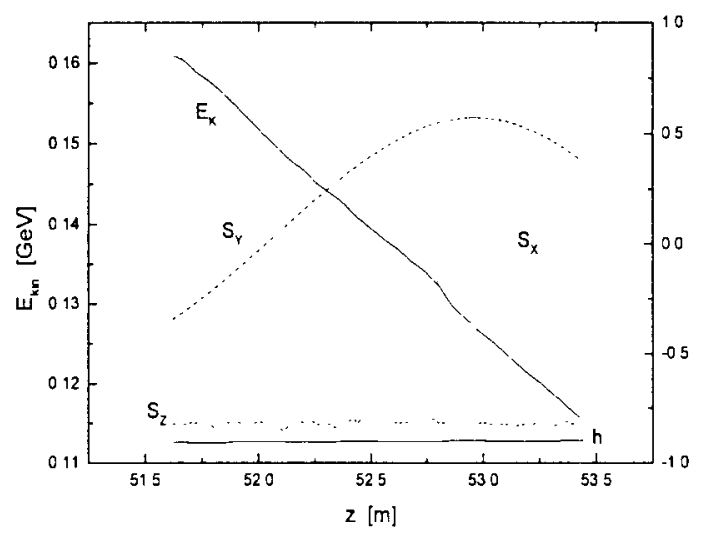

Fig. 2. Kinetic energy, spin vector components, and helicity for one particle in the liquid hydrogen minicooler.

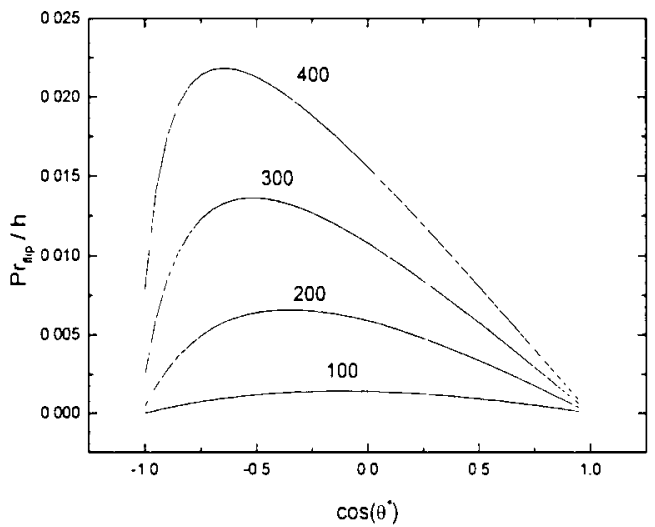

Fig. 3. First order QED calculation of the probability that a muon scattered elastically through an angle $\theta^{*}$ ends up with its spin in the opposite direction from the direction before the scattering. The probability is divided by the particle's helicity. The numbers on the curves indicate the muon LAB momentum in $\mathrm{MeV} / \mathrm{c}$.

flip probability by integrating the 1st order QED matrix element [17] over scattering angles. For a typical step size $\sim 1 \mathrm{~mm}$ in liquid hydrogen we find this probability is less than $10^{-7}$ and is thus indeed negligible.

We next consider the spin flip probability in elastic scattering events from the atomic electrons. We have computed [15] the lowest order QED matrix element for the helicity flip probability in elastic scattering. The results are shown in Fig. 3 for several values of the muon LAB momentum.

The spin flip probability vanishes in both the forward and backwards directions. For muons with a typical momentum of $200 \mathrm{MeV} / \mathrm{c}$, the maximum spin 


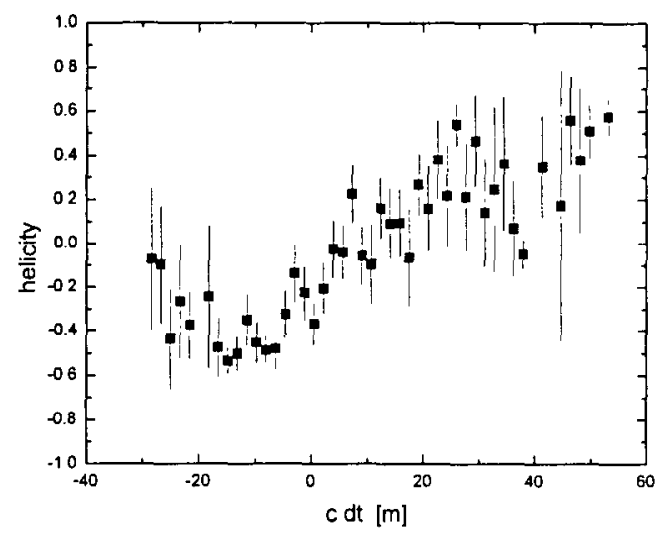

Fig. 4. Average muon helicity versus bunch position at the end of the double phase rotation system

flip probability in an elastic scattering event is $\sim 5 \times 10^{-3}$. For low momentum muons the flip probability is near 0 , in agreement with the general principle that in non-relativistic scattering the particle's spin tends to remain aligned with its initial direction.

\section{Conclusions}

We have simulated polarization effects in the $480 \mathrm{~m}$ long, double phase rotation front end for the Neutrino Factory [12]. In addition to the pion capture solenoid, rf phase rotation, and mini-cooler mentioned earlier, this system includes a long drift region, induction linac, buncher, and ionization cooling lattice. Figure 4 shows the average helicity in each bunch at the end of the system.

We see that the average bunch helicity varies from about -60 to $+60 \%$. There is a strong correlation of the strength of the helicity with its temporal location in the bunch train. However, the average helicity is not a meaningful measure for the distribution as a whole. We define instead the effective polarization

$$
P_{e f f}=\int h^{2}(t) I(t) d t
$$

where $I(t)$ is the normalized intensity at time t. The effective polarization was $0.355 \pm 0.007$ at $52 \mathrm{~m}$ into the channel (at the entrance to the minicooler, after the decay region). The effective polarization at the end of the channel was $0.350 \pm 0.015$. Thus the loss in effective polarization was only $1.4 \%$. 


\section{Acknowledgements}

We would like to thank Alain Blondel, Andy van Ginneken, and Bob Palmer for useful discussions. This work was supported by the U.S. Department of Energy under contracts DE-AC02-98CH10886 and DE-FG03-92ER40695.

\section{References}

[1] A. Blondel, Muon polarization in the neutrino factory, http://muonstoragerings.web.cern.ch/muonstoragerings/Workshop/writeup.html

[2] W. Marciano, The physics of muon colliders: a perspective, in D. Cline (ed), Physics Potential and Development of $\mu^{+} \mu^{-}$Colliders, AIP Conf. Proc. 441 (1998) 347-53.

[3] D. Perkins, Introduction to High Energy Physics, (Addison-Wesley, 1972), p.150.

[4] R. Fernow, J. Gallardo \& Y. Fukui, Muon polarization effects in the front end of the neutrino factory, Mucool note 129, 2000. This series of muon collider and neutrino factory technical notes can be found at http://www-mucool.fnal.gov/notes/notes.html

[5] S. Hayakawa, Polarization of cosmic ray mesons: theory, Phys. Rev. 108 (1957) 1533-7.

[6] M. Perl, High Energy Hadron Physics, (Wiley, 1974), p.232.

[7] J. Gallardo, R. Fernow \& Y. Fukui, A short note on the Wigner rotation, unpublished BNL Muon Technical Note MU-039, 2000.

[8] R. Fcrnow, ICOOL: a simulation code for ionization cooling of muons bcams, in A. Luccio \& W. MacKay, eds., Proc. 1999 Part. Accel. Conf., p. 3020.

[9] The $\mu^{+} \mu^{-}$Collider Collaboration, Muon-muon collider feasibility study, in New Directions for High Energy Physics, Proc. Snowmass 96, p. 432; C. Ankenbrandt et al, Status of muon collider research and development and future plans, Phys. Rev. ST-AB 2 (1999) 081001-1-73, p. 20.

[10] J.D. Jackson, Classical Electrodynamics, 3rd ed., (Wiley, 1998), p. 561-5.

[11] S.Y. Lee, Spin Dynamics and Snakes in Synchrotrons, (World Scientific, 1997), p.9-14.

[12] The case we considered was taken from http://pubweb.bnl.gov/people/palmer/nu/pjksim/icool/ as the data files existed on 25 March 2000. 
[13] B. Norum \& R. Rossmanith, Polarized beams in a muon collider, Nuc. Phys. B (Proc. Suppl.) 51A (1996) 191-200.

[14] G. Ford \& C. Mullin, Scattering of polarized Dirac particles on electrons, Phys. Rev. (1957) 477-481.

[15] R. Fernow, J. Gallardo \& Y. Fukui, On muon depolarization effects in an ionization cooling channel, Mucool note 115, 2000.

[16] M. Rose \& H. Bethe, On the absence of polarization in electron scattering, Phys. Rev. 55 (1939) 277-289.

[17] J.D. Bjorken \& S.D. Drell, Relativistic Quantum Mechanics, (McGraw-Hill, 1964), eq. 7.99 . 